\title{
Registro de la dinámica \\ témporomandibular mediante ultrasonidos con ARCUSdigma de KaVo
}

\author{
Cárdenas Martos $A^{*}$, Del Castillo Salmerón $R^{* * * *}$, Rodríguez Pérez $M^{*}$, \\ Muñoz Puerto $\mathrm{AB}^{*}$, Montero Martín J*, Ruiz Miranda $\mathrm{M}^{* * *}$, Otero Ávila A**, \\ Maroto García J****
}

\section{RESUMEN}

A lo largo del tiempo se han descrito distintos métodos para conocer y registrar la dinámica mandibular. En el presente trabajo exponemos un nuevo método basado en la emisión y recepción de ultrasonidos que nos va a permitir trabajar en tiempo real con reproducciones digitales de los movimientos que hace el paciente. Este sistema, recientemente creado por la empresa alemana KaVo, puede ser una gran ayuda, no tan solo para acelerar y mejorar el montaje y ajuste de modelos en articulador, sino para conocer en el momento, y antes de enfrentarse a un trabajo de restauración, las características de los movimientos mandibulares del paciente y poder dejarlo registrado en nuestra historia. Del mismo modo, esta posibilidad de trabajar con gráficos de movimiento mandibular en tiempo real tiene, a nuestra forma de ver, infinidad de posibilidades en el área de la educación, investigación...etc. Por tanto, el primer paso es presentar y conocer las posibilidades del ARCUSdigma ${ }^{\circledR}$ de KaVo (KaVo elektrotechnisches, Werk, GMBH, Leutkirch,Germany) .

Palabras clave: Dinámica témporomandibular, axiografía, ultrasonidos

\section{SUMMARY}

Throughout the time different methods have been described to know and to register temporomandibular dynamics . In the present work we expose a new method based on the emission and reception of ultrasounds that is going to allow us to work in real time with digital reproductions of the movements that the patient does. This system, recently created by the German company KaVo, can be a great aid, not so single to accelerate and to improve the assembly and adjustment of models in articulator, but to know in the moment, and before facing a work of restoration, the characteristics of the patient jaw movements and to leave registered it in our history. In the same way, this possibility of working with mandibular traffic diagrams in real time has, as we think, infinity of possibilities in the area of the education, investigation... etc. Therefore, the first step is to present and to know ARCUSdigma (KaVo elektrotechnisches, Werk, GMBH, leutkirch,Germany) possibilities.

Key words: Temporomandibular dynamics, axiography, ultrasounds

* Licenciado/a en Odontología por la Universidad de Granada. Alumno/a del Título de Experto en Prótesis Dental.

** Profesor colaborador de la Facultad de Odontología de la Universidad de Granada.

*** Profesor asociado de la Facultad de Odontología de la Universidad de Granada

**** Profesor titular de la Facultad de Odontología de la Universidad de Granada

Cárdenas Martos A, Del Castillo Salmerón R, Rodríguez Pérez M, Muñoz Puerto AB, Montero Martín J, Ruiz Miranda M, Otero Ávila A, Maroto García J. Registro de la dinámica témporomandibular mediante ultrasonidos con ARCUSdigma de KaVo. Av. Odontoestomatol 2003; 19-3: 131-139. 


\section{INTRODUCCIÓN}

La axiografía representa la manera más fidedigna de trabajar con dinámica mandibular, ya sea para conocer su funcionamiento $(1,2)$, evaluar los desórdenes témporomandibulares (3), o evaluar los posibles planes de tratamiento en función a la misma (4) o a otras pruebas más complejas como la sonografía articular (5). Así mismo se ha tratado de comparar el trabajo axiográfico, analógico o digital, con distintas pruebas diagnósticas como el examen clínico (6), la resonancia magnética computerizada (7), o la sonografía articular (8).

El gran inconveniente de la axiografía es su inoperancia en la clínica, debido a su dificultad de manejo, tiempo de entrenamiento (no sólo del profesional sino del paciente para el examen)... etc. En un intento de llevar a la clínica las ventajas de poder trabajar con registros dinámicos del paciente en tiempo real se ha creado ARCUSdigma ${ }^{\star}$ (KaVo elektrotechnisches, Werk, GMBH, leutkirch, Germany), un sistema que creemos, tiene importantes imbricaciones no sólo en la práctica clínica diaria, sino también en la docencia e investigación de la dinámica témporomandibular.

\section{EL SISTEMA ARCUSdigma}

ARCUSdigma es un sistema de medición y reproducción de los movimientos mandibulares basado en la transmisión de ultrasonidos. Tiene tres utilidades básicas:

- Ajuste de articulador: Como sistema para calcular, a partir de los movimientos del maxilar inferior del paciente todos los valores de ajuste necesarios para el articulador PROTAR ${ }^{\circledast}$ de KaVo. Permite calcular esos mismos valores pero ya reales, ya sea para el ajuste de cualquier otro articulador o para el estudio de dichos valores utilizando un software especial para ello.

- Análisis funcional: El sistema nos permite trabajar en tiempo real con cualquier movimiento del paciente pudiendo grabarlo, reproducirlo en los tres ejes del espacio en los puntos articular derecho, izquierdo e incisal, parar en cualquier momento la reproducción e imprimir los gráficos de dicho movimiento.

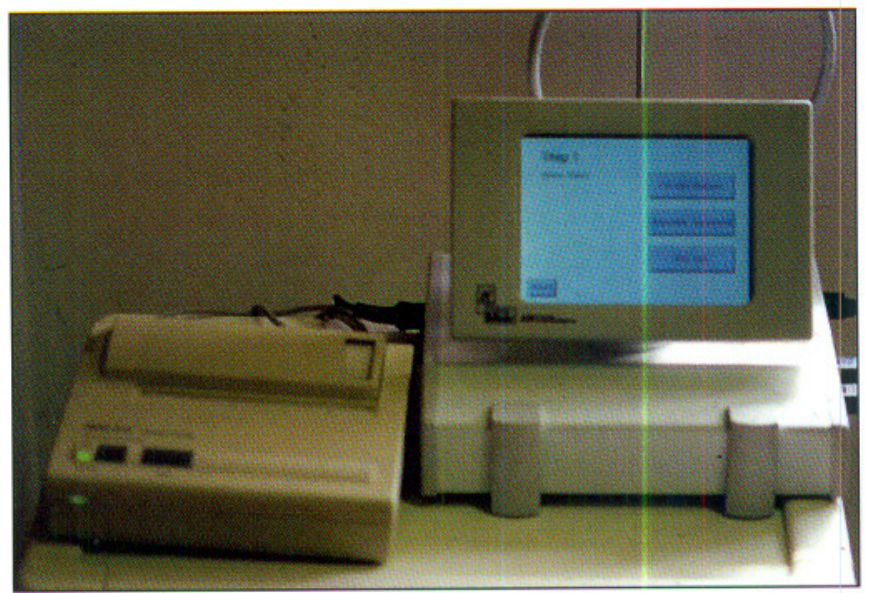

Fig. 1. El ARCUSdigma con su PU y pantalla táctil (derecha) y la impresora térmica (izquierda).

- Análisis electrónico de posición (EPA Test, Electronic Position Analysis): Gracias a este sistema podemos comparar dos posiciones articulares (Vg.- un registro de relación céntrica tomado con cera y la posición de máxima intercuspidación del paciente) y valorar la discrepancia entre ambas posiciones tanto cualitativamente (dirección) como cuantitativamente (medida en milímetros de la discrepancia).

Se compone de un aparato de control central con una pantalla TFT sensible al tacto. Además incluye un conmutador de pie para facilitar su uso en la clínica.

Puede acompañarse de una impresora térmica configurada para imprimir los informes de registros de este aparato, o bien imprimir la pantalla de trabajo en cualquier momento de la sesión (véase figura 1).

\section{El sistema de emisión-recepción de ultrasonidos se divide en dos partes:}

- Por un lado tenemos un arco superior para fijar al cráneo que se compone de dos almohadillas que se colocan detrás y encima de las orejas unidas por el arco en sí, un sistema de apoyo nasal ajustable a cada paciente y el sistema de cuatro receptores (micrófonos) de ultrasonidos que irán fijados por medio de un imán al propio arco.

- En el maxilar inferior se coloca un sistema de triple 


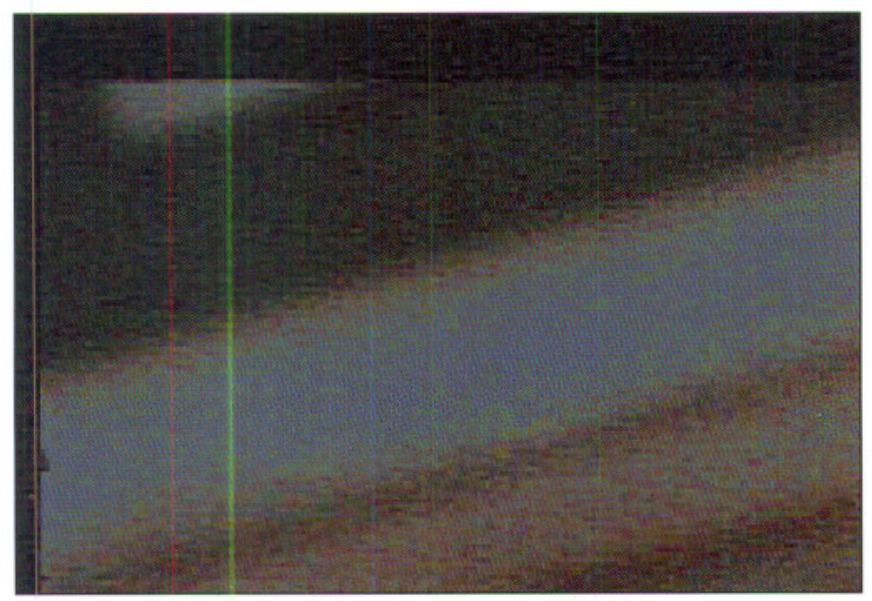

Fig. 2. El sistema de sensores y su colocación en el paciente por medio del arco superior y las horquillas inferiores.

emisión de ultrasonidos que mediante un imán se fija a una horquilla que será la que se fije en el maxilar inferior y transmita el movimiento hasta el conjunto de emisores (véase fig.2). Existen dos tipos de horquillas para el maxilar inferior:

- Una horquilla de mordida que vamos a utilizar para transportar los modelos al articulador mediante la misma con un registro de mordida y un soporte especial para el articulador PROTAR incluido en el set. La horquilla tiene grabados dos orificios identificados con los números 1 y 2. Será el propio aparato el que nos designe cual de las dos posiciones de montaje será la que utilicemos para cada paciente cuando genere el informe de montaje, como veremos posteriormente.

- Otra horquilla de menores dimensiones que se fija en vestibular de las piezas anteroinferiores directamente con un sistema de resina de clínica como Protemp ${ }^{\circledR}$ de 3M/ESPE, o cualquier otro, pudiendo ayudarse de pegamento acrílico para conseguir la adhesión necesaria para realizar las mediciones. Como vamos a tomar impresiones al paciente para su montaje en articulador también cabe la posibilidad de elaborar indirectamente la fijación con el fin de poder repetir la medición las veces que queramos, utilizando para ello el mismo tipo de resinas o cualquier otro de los que se emplean usualmente en un laboratorio dental para elaboración de cubetas, provisionales... etc. Si el paciente tuviese una sobremordida que imposibilitara la fijación a nivel anteroinferior de la horquilla se puede alar- gar con la misma resina para fijarse en labial de premolares y molares.

\section{Principios de funcionamiento del ARCUSdigma:}

El mecanismo de funcionamiento del ARCUSdigma es bastante sencillo. Una vez colocado el arco de fijación superior y el soporte inferior, al conectar el sistema y empezar a trabajar la parte fijada a la mandíbula comenzará a emitir ultrasonidos mientras que la parte superior los recibirá. De forma que, al recibir cada uno de los micrófonos ultrasonidos procedentes de los tres emisores se crea un polígono espacial (véase figura 3) creado por los doce segmentos de medición (de los tres emisores a cada uno de los cuatro receptores) cuya morfología recoge el sistema y transforma en la información que le estemos pidiendo (Vg.- trayectoria condílea a partir de los movimientos de apertura y protrusión).

En cuanto a la mecánica de trabajo con el ARCUSdigma lo único que nos va a requerir es seguir un protocolo de trabajo establecido por el propio aparato en una secuencia lógica e intuitiva.

La pantalla principal del ARCUSdigma nos da a elegir entre las tres funciones antes mencionadas:

- Análisis funcional

- Ajuste de articulador

- Análisis electrónico de posición (EPA Test).

- Posicionamiento de modelos (arco facial electrónico): Para realizar cualquiera de las tres funciones lo primero que hemos de hacer, tras haberle colocado al

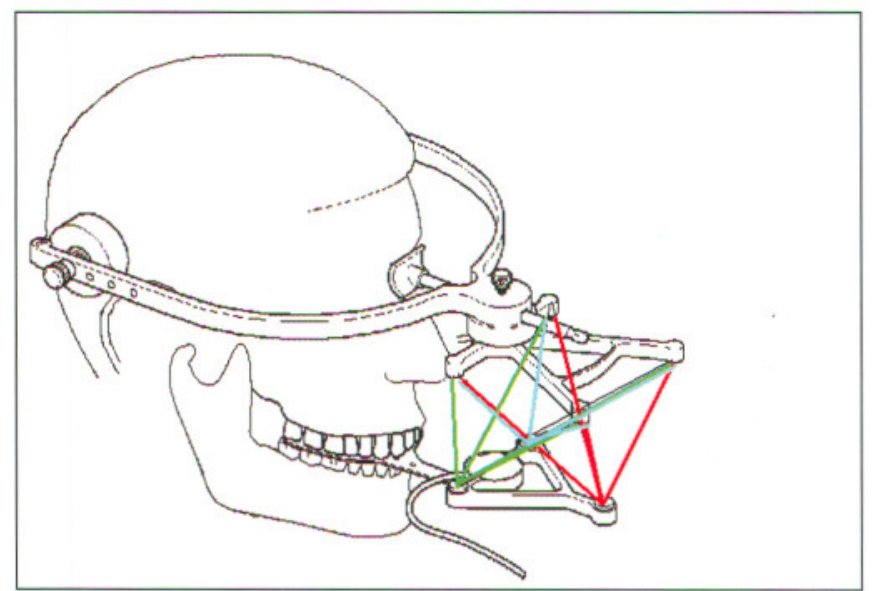

Fig. 3. Segmentos de medición de ultrasonidos en el ARCUSdigma durante su funcionamiento. 


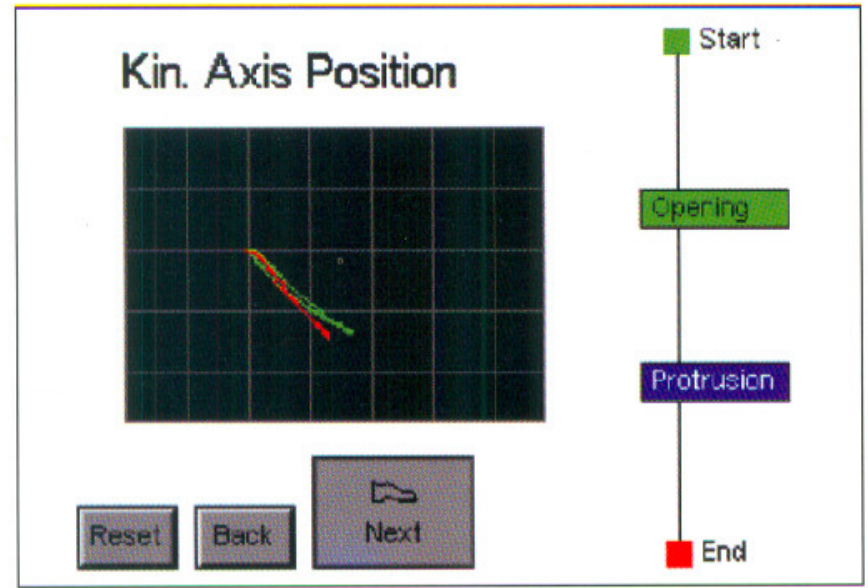

Fig. 4. Movimientos de apertura y protrusión para localización de eje cinemático.

paciente las fijaciones superior e inferior será la determinación de la posición de los modelos, como con cualquier arco facial pero con la facilidad de realizarlo electrónicamente:

Para ello primero colocaremos la horquilla de mordida y haremos morder al paciente mientras presionamos el pedal o la pantalla del ordenador. Cuando el ordenador haya determinado la posición del maxilar superior nos permitirá proseguir, colocando la horquilla de fijación anteroinferior, y fijando a esta los emisores (con la precaución de colocarlos correctamente mirando hacia los receptores). Volvemos a pisar el pedal o pulsar en la pantalla y cuando el ordenador haya fijado la posición nos volverá al menú avanzando a la siguiente parte.

- Registro del eje cinemático:

Otra función común, pero tan solo a los exámenes de análisis funcional y prueba EPA será el registro del eje cinemático. Para ello, con la horquilla que ya tenemos colocada en el paciente le pedimos a este que nos haga un movimiento de apertura mientras pulsamos el pedal y soltamos al final de éste. Posteriormente le pedimos un movimiento de protrusión pulsando nosotros a la vez el pedal o pantalla.

En la pantalla del ordenador veremos la reproducción gráfica de ambos movimientos (véase figura 4) de la que, en algunos casos podremos sospechar algún diagnóstico (por la presencia de saltos, interrupciones, discontinuidad en los movimientos...). No importa si el maxilar inferior rota o lateraliza en la

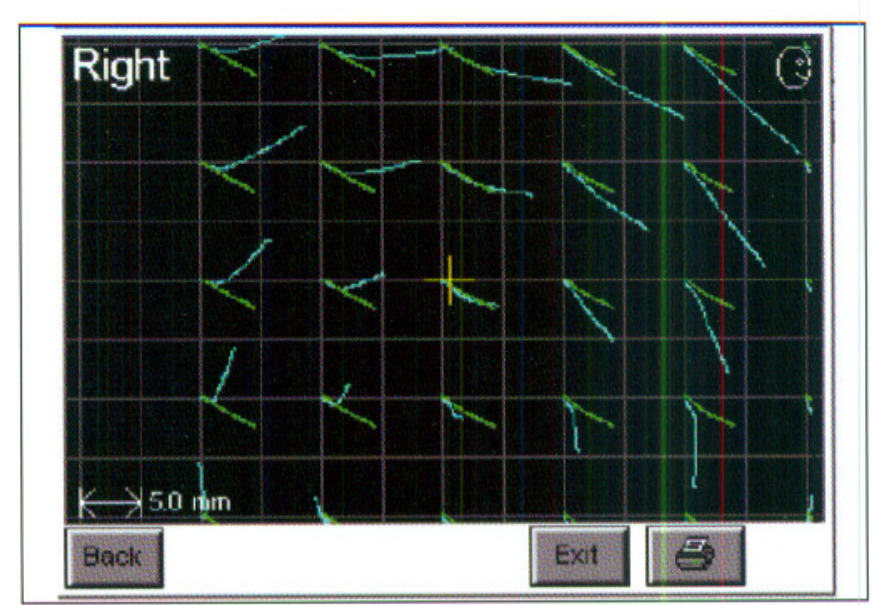

Fig. 5. Seleccionando el eje cinemático para la articulación derecha.

apertura, el ordenador trabaja en el plano sagital para obviar estos movimientos, evitando así errores por otros puntos de eje teóricamente posibles.

Una vez aceptados los dos movimientos nos lleva a una pantalla dividida en segmentos de $5 \mathrm{~mm}$. El aparato ha calculado automáticamente el punto de eje cinemático (para la articulación derecha) y lo reproduce en el centro de la pantalla (véase figura 5), y alrededor a una distancia de $5 \mathrm{~mm}$ presenta mas puntos de eje posibles (en realidad no son buenas elecciones porque el ordenador ya ha elegido automáticamente el punto en el que mas coincide apertura y protrusión y lo ha colocado en el centro de la pantalla, pero de cualquier manera nos permite que elijamos aquel que el clínico perciba como mejor). Si no intervenimos de forma activa el ordenador entiende que estamos de acuerdo con su elección y nos presentara la misma pantalla pero para la articulación izquierda.

$\mathrm{Si}$, por el contrario eligiésemos otro eje cinemático distinto del que ha preseleccionado el aparato bastaría con tocarlo en la pantalla para que el ordenador genere una nueva reproducción de ejes cinemáticos, esta vez a intervalos de $2,5 \mathrm{~mm}$ donde coloca en el centro el seleccionado por nosotros previamente y alrededor otras opciones. Ya seleccionemos el eje que sea al tocarlo, tendremos que volver a elegir eje pero esta vez en reproducciones a un intervalo de $1,5 \mathrm{~mm}$. Una vez seleccionado este eje repetiríamos el procedimiento para la articulación opuesta (izquierda).

Esta función nos puede permitir, en casos de análisis funcional o pacientes con disfunción témporomandi- 
bular, escoger, basándonos en la reproducción sagital de los movimientos de apertura y cierre, aquel eje en el que exista menor discrepancia entre ambos movimientos, ya sea con fines diagnósticos, terapéuticos o investigadores.

\section{- Análisis funcional:}

Una vez correctamente posicionados los modelos y elegido el eje cinemático para cada articulación podemos realizar el análisis funcional del paciente.

Para ello aparece una pantalla como se describe a continuación (véase figura 6):

- En su parte superior se nos ofrecen tres vistas de los movimientos que por defecto son articular sagital izquierda, incisal frontal y articular sagital derecha. Pueden cambiarse una o más simplemente tocando la cabeza que nos ilustra la posición, pudiendo poner vista horizontal o cambiar cualquiera de las anteriores en el orden que queramos. Lo que no podemos cambiar es que las dos laterales corresponden a cada una de las articulaciones y la central al punto incisal. Las rejillas que aparecen en cada una de las pantallas reflejan espacios reales de $5 \mathrm{~mm}$ para las dos pantallas articulares, y de $10 \mathrm{~mm}$ para la reproducción incisal central como se refleja en la parte inferior de cada una de las pantallas.

- Debajo y a la izquierda de las tres pantallas aparece el icono del sistema de emisores como indica-

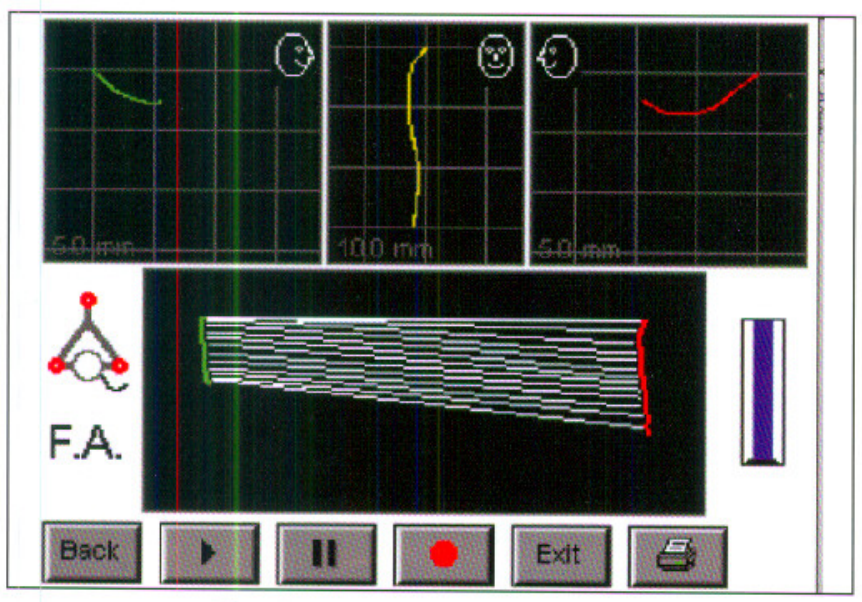

Fig. 6. Pantalla de análisis funcional. dor de control: cuando dicha figura aparezca en rojo significa que no se esta realizando medición, y cuando aparezca en verde será porque estemos midiendo ese movimiento (estará el pedal pulsado o la tecla del circulo rojo de la pantalla). Justo debajo aparecerán las siglas F.A. que nos indican que estamos dentro del análisis funcional.

- En la parte central aparece una pantalla de la vista horizontal por encima de las dos articulaciones; desde este punto de vista podemos apreciar la sincronía de movimientos entre ambas. En éste habrá partes en las que alguna de las articulaciones se decelera "estorbando" o ralentizando el movimiento de la otra, lo cual se refleja con trazos no paralelos mientras que las que sean totalmente paralelas indican movimientos en los que no hay "estorbo" o molestia por parte de ninguna de las articulaciones.

- En la parte central y derecha de la pantalla hay otro indicador en forma de barra que nos sirve para conocer el estado de saturación de la memoria, que cuenta con unos 10-12 segundos de tiempo de memorización. Todos los movimientos memorizados se pueden repetir tantas veces como se desee tan solo tocando la tecla triangular (REPLAY).

- La parte inferior de la pantalla esta ocupada por una serie de teclas de función meramente intuitiva que, por enumerar de izquierda a derecha son:

Tecla BACK - volveremos al menú anterior

Tecla (REPLAY) - reproducimos, tantas veces como deseemos el movimiento registrado. Cuando pedimos más de una vez que reproduzca el mismo movimiento, por defecto va cambiando las distintas vistas del mismo.

Tecla II (PAUSE) - en cualquier momento de la repetición podemos pararla y volver al principio de la misma.

Tecla • (REC) - tiene la misma función que el pedal y nos va a permitir memorizar en el ARCUSdigma los movimientos realizados por el paciente.

Tecla EXIT - salimos del análisis funcional.

Tecla PRINT - imprimimos la pantalla con los gráficos de movimiento.

Realizar el análisis funcional consistirá en ir pidiendo al paciente que realice los movimientos que deseemos 


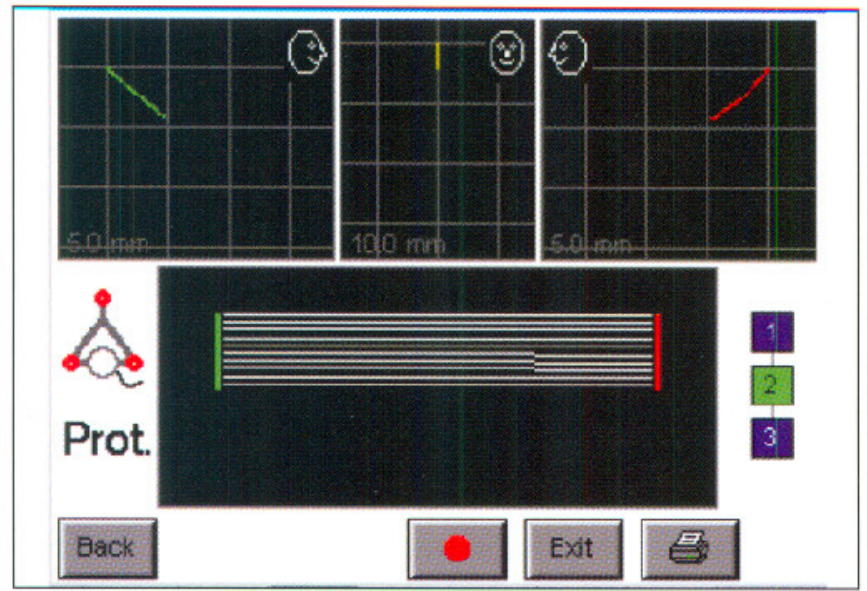

Fig. 7. Registros de protrusiva para montaje en articulador.

ver reflejados en la pantalla sincronizándonos con éste para darle a la vez al botón REC o pisar el pedal permitiendo así que el sistema memorice el movimiento. De esta forma podemos realizar un análisis de cualquier movimiento del paciente y verlo reflejado en la pantalla en los tres ejes del espacio, dándonos la herramienta básica de enseñanza e investigación en dinámica témporomandibular. En esta función de análisis nos deja el sistema que seamos los profesionales los que vayamos registrando los distintos movimientos sin imponernos ninguna norma ya que es el propio análisis el fin del mismo, es decir, que una vez hechos todos los movimientos que deseemos salimos del menú o imprimimos los resultados terminando así el análisis funcional.

\section{- Ajustes para montaje del articulador PROTAR:}

Paso previo como para las demás funciones del ARCUSdigma será el posicionamiento de modelos, si ya lo hubiese realizado pasaría automáticamente al siguiente paso.

El segundo paso sería los movimientos de protrusión / retrusión. Volveríamos a entrar en una pantalla similar a la del análisis funcional (véase figura 7) con la diferencia de que ahora debajo del icono de emisores aparece la palabra "Prot.". El sistema necesita que el paciente realice tres movimientos de protrusión (ha cambiado la barra central-derecha por un visor con los números del 1 al 3 para indicarnos cual es el movimiento que estamos registrando) que reflejará en la pantalla igual que en el apartado anterior.
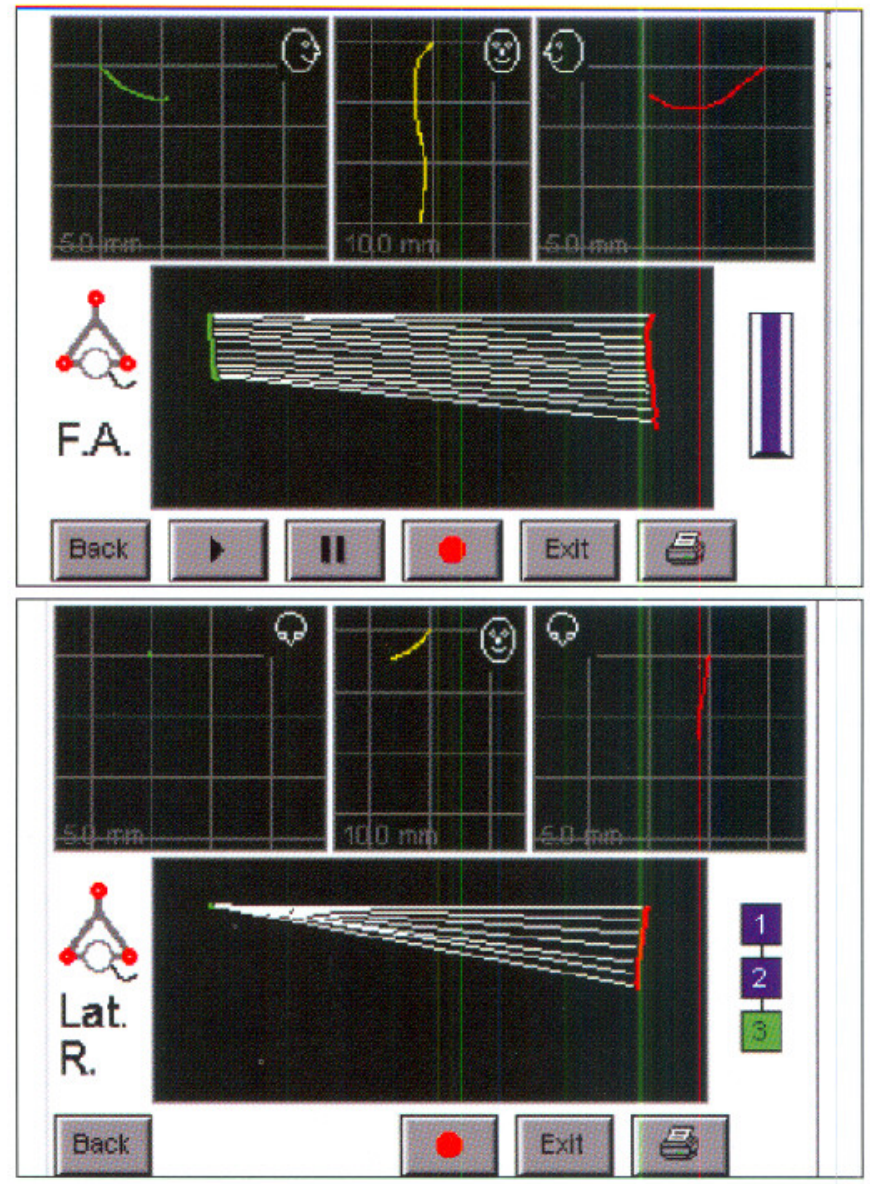

Fig. 8. Ejercicio de laterotrusión derecha para montaje en articulador.

Estos movimientos han de ser deslizantes bajo contacto dentario.

Para grabar los movimientos habrá que pisar el pedal mientras el paciente los realiza, estos han de ser al menos de 4,5 mm para que el ordenador los de por válidos (podemos ayudar al paciente manipulándolo para llegar al final de dicho movimiento). Con el valor medio de las tres protrusiones el aparato nos calculará la trayectoria condílea del paciente así como características frontales de la guía incisal.

Después tendríamos que registrar la laterotrusión derecha de la misma manera que antes (véase figura 8). En este tipo de movimientos es conveniente completar el movimiento entero hasta los límites del mismo, y por tanto, ayudar al paciente por medio de una manipulación ligera.

El registro de la laterotrusión izquierda es idéntico al registro de la laterotrusión derecha.

Una vez que el aparato ha dado por válidos todos los 


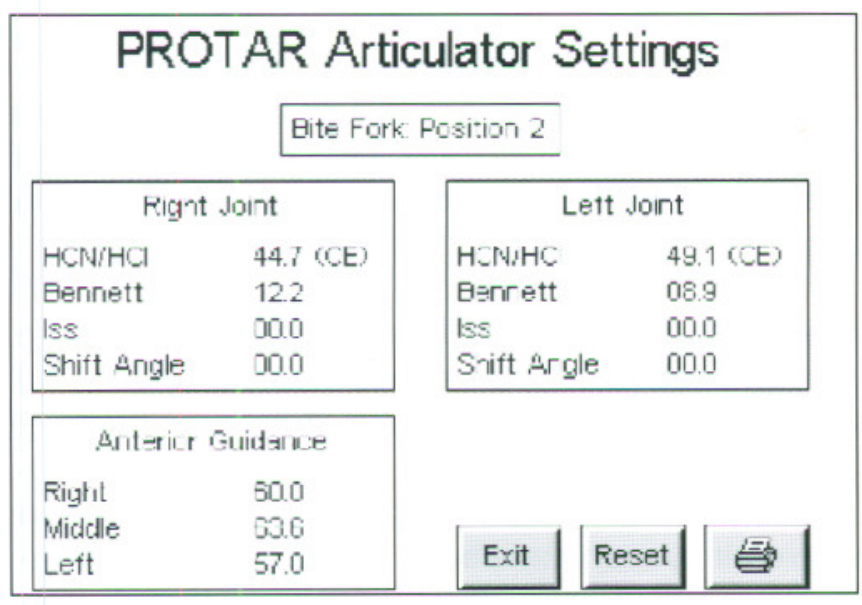

Fig. 9. Informe para montaje en articulador.

movimientos nos va a dar un informe para ajustar el montaje en el articulador PROTAR en el que se incluye (véase figura 9):

\section{1.- Posición de la horquilla de mordida:}

Como ya comentamos anteriormente existen dos orificios en la horquilla de posicionamiento del maxilar superior identificados con los números 1 y 2 (véase figura 10). A la hora de realizar el montaje nos aseguraremos que el orificio que esté colocado en el aditamento para montaje sea el designado por el aparato.

2.- Articulación izquierda / derecha:

- $\mathrm{HCN} / \mathrm{HCI}$ (CE) - inclinación de la trayectoria condílea (medida para plano de Camper externo o cutáneo)

- Bennett - ángulo de Bennett

- Iss - Movimiento lateral inmediato (Inmediate side shift)

- Shift Angle - ángulo del movimiento lateral inmediato. Necesitamos un aditamento especial para poder reflejarlo en el articulador (Supl. Shift angle para PROTAR 7 de KaVo)

\section{3.- Guía anterior:}

Los valores que nos da han de reflejarse en otro aditamento especial que no viene de serie en el PROTAR 7 (Supl. Anterior guidance para PROTAR 7 de $\mathrm{KaVo})$

- Izquierda

- Media

- Derecha

La desviación estándar para las medidas de montaje

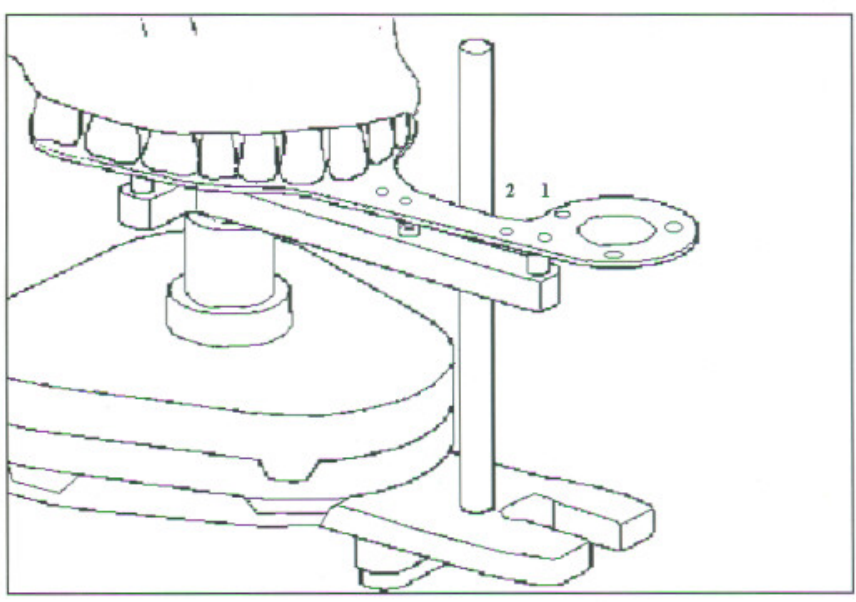

Fig. 10. Posiciones de acoplamiento de la horquilla para maxilar superior: posición 1 (celeste), posición 2 (rojo).

para el PROTAR 7 de KaVo con ARCUSdigma es de 2 grados.

\section{- Prueba de Análisis de Posición Electrónico:}

Al igual que antes, si ya hemos determinado las posiciones de los maxilares y el eje cinemático, entraremos directamente en la pantalla del test EPA. En esta función vamos a poder comparar dos posiciones del maxilar inferior.

Para ello contamos con una pantalla en la que tenemos:

- En la parte superior una barra coloreada que nos marcará el grado de discrepancia entre ambas medidas. A su izquierda tenemos el marcador de control de grabación ya visto (icono del emisor) y a la derecha nos dice que estamos en la pantalla del EPA (véase figura 11).

- En el centro hay dos pantallas divididas en círculos concéntricos separados con correspondencia a una distancia de 0,3 $\mathrm{mm}$, para la articulación derecha (R) y la izquierda (L). Aquí veremos los movimientos de ambas articulaciones en el plano sagital.

- En la parte inferior algunas de las mismas teclas que vimos en el menú de análisis funcional, en concreto las teclas BACK, REC, EXIT y PRINT.

El funcionamiento de esta prueba es bastante sencillo. Queremos comprobar, por ejemplo, que tenemos una posición de "relación céntrica" es decir repetible. Pues bien, ponemos al paciente en dicha 


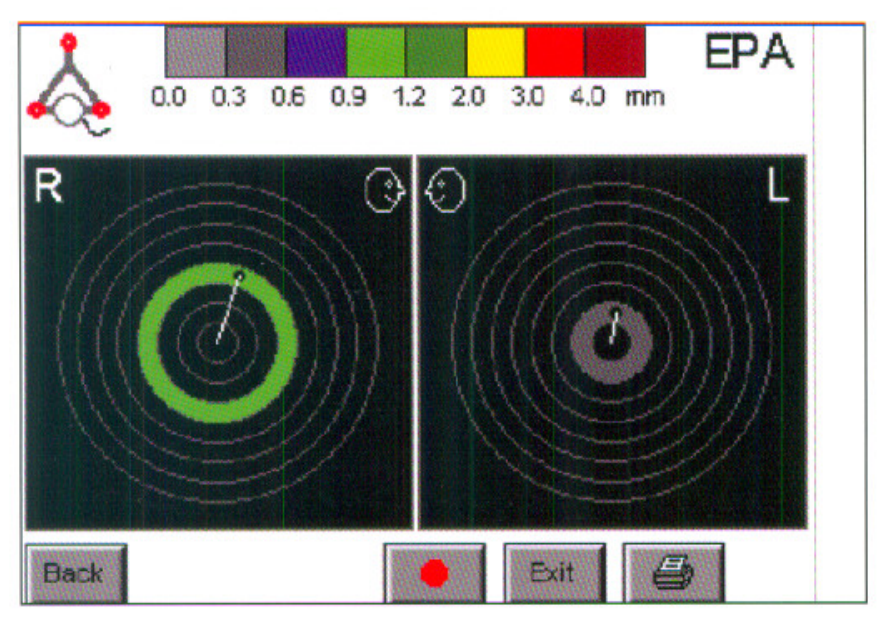

Fig. 11. Pantalla de la prueba análisis de posición electrónico (test EPA).

posición y pulsamos el conmutador de pie o la tecla REC de la pantalla, ARCUSdigma guarda dicha posición en su memoria. Tomamos un registro de céntrica con cera dura (tipo Moyco) pero queremos asegurarnos que es la misma posición que registramos en un principio, así que colocamos la galleta y hacemos que el paciente la muerda en la posición marcada. Una vez colocada pulsamos de nuevo el conmutador y cuando soltemos el ordenador nos indicará si existe desviación respecto al primer movimiento que grabamos y en que grado y en que articulación existe mayor desviación. Para ello aparece una línea blanca en la dirección de la desviación y se colorea el círculo concéntrico con el color correspondiente a la cantidad de desviación registrada (puede anotarse sirviéndose de la regla situada en la parte superior de la pantalla):

Gris claro - hasta 0,29 $\mathrm{mm}$ de desviación

Gris oscuro - de 0,3 a 0,59 mm de desviación

Azul - de 0,6 a 0,89 mm de desviación

Verde pistacho - de 0,9 a 1,19 mm de desviación

Verde pino - de 1,20 a 1,99 mm de desviación

Amarillo - de 2 a 2,99 mm de desviación

Naranja - de 3 a 3,99 mm de desviación

Granate - de $4 \mathrm{~mm}$ de desviación en adelante.

La desviación estándar para las medidas de la prueba EPA está en una décima de milímetro $(0,1 \mathrm{~mm})$.

Así podemos, entre otras cosas, comprobar que los registros que llevamos a un articulador son coincidentes con la posición que queríamos reflejar, o ver si una férula nos lleva a la posición que deseamos que nos lleve o modificarla para que lo haga.
- Trabajando con el ARCUSdigma de KaVo:

El tiempo de trabajo para tomar las medidas de ajuste para montar el articulador PROTAR es realmente corto, si excluimos el tiempo que nos llevará fijar la horquilla paraoclusal, por supuesto.

Resulta de gran utilidad llevar el procedimiento de medida a través de una pantalla sensible al tacto, así como la posibilidad de manejar el aparato desde el conmutador de pie.

La función de análisis de posición electrónico nos va a permitir, fundamentalmente comparar cuantitativamente varios registros de céntrica entre sí, o en relación con cualquier otra posición como máxima intercuspidación u otra posición repetible por el paciente que hayamos registrado. Aun más, mediante el EPA podremos evaluar las férulas oclusales en relación a la posición mandibular a la que nos esta llevando (o que deberían llevarnos).

En cualquiera de las funciones nos permite ver los movimientos en los tres ejes del espacio. Así mismo, cualquier movimiento que hayamos grabado en el aparato, podemos reproducirlo tantas veces como queramos, empezando y parando la reproducción en el momento que deseemos.

Existe un software adicional de análisis funcional que nos permitiría examinar los movimientos de la mandíbula buscando alteraciones de los mismos en cualquier punto de su trayecto tridimensionalmente. Considerándolo útil por tanto, para un análisis funcional previo y necesario para solventar los problemas prostodónticos y restauradores extensos, para los que, con otros sistemas clásicos necesitaríamos mayor tiempo, y preparación para realizarlo. Sin embargo, con este aparato podemos observar simultáneamente los movimientos condilares de ambas articulaciones, la forma del arco cinemático y su trabajo en la dinámica témporomandibular, buscando directamente indicios que nos conduzcan hacia diagnósticos de limitación de movimientos, incoordinación articular, o hipermovilidad. La consideración de las causas de estos problemas (artrológicas, miológicas u oclusales) pueden ser consideradas después, pero su consecuencia, la limitación fisiológica, ya ha sido reflejada en nuestra historia.

La pantalla incisal central nos va a permitir ver en 
una vista frontal diagramas del movimiento mandibular que ya conocemos en los distintos planos (sagital - Posselt; frontal, horizontal o de Gyssi), pudiendo, de nuevo, evaluar el comportamiento dinámico acelerando o decelerando la reproducción de dichos diagramas.

En resumen esta nueva herramienta tiene una serie de ventajas que podemos enumerar:

- Programación de los datos del paciente en el articulador (PROTAR de $\mathrm{KaVo}$ ) en menos de cinco minutos. Algunos lo llaman el arco facial electrónico, pero pensamos que es mucho más que eso.

- Nos permite trabajar con medidas y cálculos a partir del eje cinemático.

- Manejo intuitivo y fácil a través de la pantalla sensible al tacto o el pedal.

- No necesita ninguna precalibración sobre el paciente

- Nos da la posibilidad de trabajar a través del ordenador personal (interfaz de puerto paralelo) pero no la impone, se trabaja igual con la propia CPU del aparato y se le puede conectar una impresora térmica específicamente configurada para el ARCUSdigma para imprimir los resultados.

- El software que podemos adquirir por separado para trabajar en el ordenador puede renovarse constantemente a través de Internet.

- Aunque la CPU pese 2,6 Kg, los sensores que se le colocan sobre la horquilla que sostiene el paciente tan solo pesan $22 \mathrm{~g}$.

- El principio de medición a partir de ultrasonidos obvia cualquier radiación electromagnética, por lo que no interfiere en sus medidas ninguno de los aparatos que normalmente están encendidos en nuestras clínicas (sillón, equipo de música, luces, teléfonos móviles...)

- La precisión en las medidas del test EPA es de 0,1 $\mathrm{mm}$, y para el cálculo de ángulos para el montaje en articulador PROTAR es de 2 grados. Son errores de medición conocidos, a diferencia del trabajo usual con registros en cera que no sabemos qué precisión tienen, por lo que con ARCUSdigma trabajamos con medidas de intervalo muy estrecho.

\section{BIBLIOGRAFÍA}

1. Slavicek R. Clinical and instrumental functional analysis for diagnosis and treatment planning. Part 5. Axiography. J-Clin-Orthod 1988; 22 (10): 656-67.

2. Slavicek R. Clinical and instrumental functional analysis for diagnosis and treatment planning. Part 7. Computer-aided axiography. J-Clin-Orthod 1988; 22 (12): 776-87.

3. Piehslinger E, Ertl L. Computerized axiography for standardized evaluation of TMJ function and dysfunction. Medinfo 1995; 8 Pt 2:1303-4.

4. Piehslinger E, Bigenzahn W, Celar A, Slavicek R. The effect of occlusal splint therapy on different curve parameters of axiographic TMJ tracings. Cranio 1995; 13 (1): 35-41.

5. Moritz M, Behr $M$, Held $P$, Dammer R, Niederdellmann $\mathrm{H}$. Comparative study of results of electronic axiography with results of magnetic resonance imaging including $M R I$-assisted splint therapy. Acta-Stomatol-Belg 1995; 92 (1): 35-8.

6. Bernhardt O, Schwahn B, Meyer G. Craniomandibular disorders-comparative investigations with clinical examination and electronic axiography. Anat-Anz 1999; 181 (1): 51-3.

7. Piehslinger E, Schimmerl S, Celar A, Crowley C, Imhof $\mathrm{H}$. Comparison of magnetic resonance tomography with computerized axiography in diagnosis of temporomandibular joint disorders. Int-J-Oral-Maxillofac-Surg 1995; 24 (1 Pt 1): 13-9.

8. Bracco P, Deregibus A, Piscetta R, Giaretta G A. TMJ clicking: a comparison of clinical examination, sonography, and axiography. Cranio 1997; 15 (2): 121-6.

\section{CORRESPONDENCIA}

Alejandro Cárdenas Martos
c/ Blas Infante $12,2^{\circ} \mathrm{F}$
Granada 18008
Tlfno: 655517429
e-mail: dralexcardenas@hotmail.com 